\title{
DY-9760e, a Calmodulin Antagonist, Reduces Brain Damage after Permanent Focal Cerebral Ischemia in Cats
}

\author{
Masunobu Sugimura, Hideo Takamori, Hiromichi Fukushi, Yutaka Kitano, Yoshito Kanazawa, and \\ Yasufumi SHIRASAKI* \\ New Product Research Laboratories II, Daiichi Pharmaceutical Co., Ltd.; 1-16-13, Kitakasai, Edogawa-ku, Tokyo \\ 134-8630, Japan. Received October 29, 2004; accepted January 11, 2005; published online January 21, 2005
}

\begin{abstract}
DY-9760e (3-[2-[4-(3-chloro-2-methylphenyl)-1-piperazinyl]ethyl]-5,6-dimethoxy-1-(4-imidazolylmethyl)-1Hindazole dihydrochloride 3.5 hydrate), a calmodulin antagonist, provides protection against $\mathrm{Ca}^{2+}$ overload-associated cytotoxicity and brain injury after cerebral ischemia in rats. In this study, we assessed the effect of DY$9760 \mathrm{e}$ on ischemic infarct volume in cats subjected to permanent focal cerebral ischemia. DY-9760e was infused for $6 \mathrm{~h}$, beginning $5 \mathrm{~min}$ after occlusion of the middle cerebral artery. The infarct volume was measured at the end of drug infusion. DY-9760e, at the dose of 0.25 but not $0.1 \mathrm{mg} / \mathrm{kg} / \mathrm{h}$, significantly reduced cerebral infarct volume without affecting any physiological parameters, and its protective effect was mainly evident in the cerebral cortex, where the penumbra, a salvageable zone, exists. The present study demonstrates that DY-9760e protects against brain injury after focal ischemia in a gyrencephalic animal as well as in the rodents reported previously and suggests its therapeutic value for the treatment of acute stroke.
\end{abstract}

Key words DY-9760e; calmodulin; focal ischemia; gyrencephalic model; cerebral protection

Although the cellular pathophysiological mechanisms underlying ischemic brain damage remain to be fully clarified, accumulating evidence suggests that an excessive elevation of intracellular $\mathrm{Ca}^{2+}$ in neurons is a primary mechanism by which cells become injured following cerebral ischemia. ${ }^{1-3)}$ An overload of $\mathrm{Ca}^{2+}$ in neurons disrupts the ionic balance and activates various $\mathrm{Ca}^{2+}$-dependent enzymes. Treatment of ischemic animals with compounds that block $\mathrm{Ca}^{2+}$ entry through $\mathrm{Ca}^{2+}$-permeable channels has been shown to provide protection against the consequences of ischemia in laboratory animals, ${ }^{4-6}$ but these treatments have not been found suitable for use in humans. ${ }^{7-9)}$

Calmodulin is a major $\mathrm{Ca}^{2+}$-binding protein found mainly in the central nervous system. ${ }^{10,11)}$ Since many of the pathways through which $\mathrm{Ca}^{2+}$ acts involve $\mathrm{Ca}^{2+}$ /calmodulin signaling systems, including calmodulin-dependent enzymes, inhibition of calmodulin may be a possible alternative approach towards the treatment and prevention of post-ischemic injury. In fact, it has been reported that $\mathrm{Ca}^{2+}$-bound calmodulin, which is an active form, is increased following ischemia and regional changes correlate with regions of ischemic neuronal damage. ${ }^{12)}$ Furthermore, calmodulin antagonists protect cultured neurons from cell death induced by glutamate, an excitatory amino acid, and protect hippocampal CA1 neurons against hypoxia/hypoglycemia in organotypic cultures. ${ }^{13,14)}$

DY-9760e (3-[2-[4-(3-chloro-2-methylphenyl)-1-piperazinyl]ethyl]-5,6-dimethoxy-1-(4-imidazolylmethyl)- $1 H$ - indazole dihydrochloride 3.5 hydrate) is a calmodulin antagonist that is 2 to 70 times more potent than W-7, a well-known calmodulin antagonist, and can protect neurons against neuronal cell death elicited by $\mathrm{Ca}^{2+}$ overload. ${ }^{15-17)}$ This compound ameliorates brain injury that occurs after transient or permanent focal ischemia and microembolization in rats. ${ }^{18-22)}$ Although these studies in various rodent models show a hopeful characteristic of DY-9760e, it is subsequently important to assess if it exerts a protective effect in a large animal model of focal cerebral ischemia. Cats, a gyrencephalic species in common with humans, have long been used both in pathophysiological studies for cerebral ischemia and in investigations for the protective efficacy of agents. ${ }^{23-27)}$ Therefore, the present study was designed to evaluate the potential acute therapeutic effect of DY-9760e on ischemic injury resulting from permanent middle cerebral artery (MCA) occlusion in cats.

\section{MATERIALS AND METHODS}

Animals Twenty-three male Ico: Fec Eur (Tif) cats (IFFA-CREDO, L'arbresle, France), weighing 2.7 to $3.3 \mathrm{~kg}$, were used in this study. The animals were maintained on canned food (CK, Oriental Yeast, Tokyo, Japan), solid food (NK-C, Nihon Nosan, Yokohama, Japan) and tap water, which were given once a day under a constant 12-h dark-light cycle. All experimental procedures were performed in accordance with the in-house guidelines of the Institutional Animal Care and Use Committee of Daiichi Pharmaceutical. All efforts were made to minimize animal suffering and to reduce the number of animals used.

MCA Occlusion Model in Cats Each cat was anesthetized with ether, intubated and immobilized with gallamine triethiodide (1 mg/kg i.v., Flaxedil, May \& Baker, Dagenham, England). Anesthesia was maintained with $1.5 \%$ halothane in a mixture of $70 \%$ nitrous oxide and $30 \%$ oxygen until the end of the investigation. All animals were mechanically ventilated with a respirator (SN-480-6, Shinano, Tokyo, Japan) to maintain a constant respiratory status throughout the experimental period. The head of the cat was placed in a stereotaxic frame (SN-1N, Narishige, Tokyo, Japan) and the left MCA was exposed by a transorbital approach with the use of microsurgical techniques. ${ }^{28)}$ In brief, the left orbit was exenterated, and the orbital roof and optic foramen were removed with a dental drill to expose the dura mater overlying the MCA. Under a surgical microscope (Type M, Konan, Hyougo, Japan), the dura was incised and the MCA was exposed. After dissection of arachnoid from the MCA, the MCA trunk was occluded with an aneurysm clip (Fine Science Tools, British Columbia, Canada). 
Measurement of Physiological Parameters Femoral arteries were cannulated bilaterally to monitor arterial blood pressure (AP-601G and WT-645G, Nihon Kohden, Tokyo, Japan) and obtain arterial blood samples for blood gas determination. Blood gases and $\mathrm{pH}$ were monitored just before and at $1 \mathrm{~h}, 3 \mathrm{~h}$ and $6 \mathrm{~h}$ after the MCA occlusion by using a blood gas system (278 Blood Gas System, Chiba Corning, Tokyo, Japan). $\mathrm{PCO}_{2}$ was kept at $33 \pm 5 \mathrm{mmHg}$ by adjusting the stroke volume of the respirator. Body temperature was measured rectally and maintained constant at $37 \pm 1^{\circ} \mathrm{C}$ with a body temperature controller (ATB-1100, Nihon Kohden, Tokyo, Japan).

Electroencephalogram Recording The electroencephalogram (EEG) was monitored to verify a unilateral reduction in brain electrical activity during occlusion. Screw electrodes were implanted into the skull on the surface of the bilateral middle ectosylvian gyri, where blood is supplied mainly by the MCA, and EEG was recorded with an electroencephalograph (EEG-4414, Nihon Kohden, Tokyo, Japan). To standardize the degree of ischemic insult during the MCA occlusion, animals in which mean ipsilateral EEG amplitude decreased to less than $30 \%$ of the preocclusion level immediately after the onset of ischemia and did not recover during the first $5 \mathrm{~min}$ of ischemia were used for evaluation. In this study, 5 of 23 cats that did not meet this criterion were excluded from further study: 3 animals did not have a $70 \%$ reduction in EEG amplitude after MCA occlusion, and 2 animals recovered EEG amplitude within 5 min after occlusion.

Administration of DY-9760e DY-9760e was synthesized at Daiichi Pharmaceutical (Tokyo, Japan) and dissolved in $50 \mathrm{~mm}$ Sörensen buffer ( $\mathrm{pH} 4.5$ ), which was prepared from $50 \mathrm{~mm}$ glycine and sodium chloride adjusted for $\mathrm{pH}$ with $1 \mathrm{~N} \mathrm{HCl}$. The right femoral vein was cannulated to administer DY-9760e. Intravenous infusion of vehicle $(50 \mathrm{~mm}$ Sörensen buffer, $3 \mathrm{ml} / \mathrm{kg} / \mathrm{h})$ or DY-9760e $(0.1$ or $0.25 \mathrm{mg} / \mathrm{kg} / \mathrm{h})$ was initiated $5 \mathrm{~min}$ after MCA occlusion via the femoral vein using an infusion pump (STC-531, Terumo, Tokyo, Japan) and continued for $6 \mathrm{~h}$. We have also confirmed that the vehicle has no effect on physiological parameters including blood pressure, blood gasses and hematocrit. ${ }^{18,21)}$ Animals were assigned to treatment groups in a random manner.

Evaluation of Infarct Volume Animals were sacrificed after a $6 \mathrm{~h}$-infusion of drug with an excess dose of pentobarbital sodium (Somnopentyl, Kyouritsu Shouji, Tokyo, Japan), and the brain was removed rapidly. The brain was coronally sectioned in nine 4-mm-thick slices with a brain slicer (Muromachi Kikai, Tokyo, Japan). The sections were numbered from 1 to 9 starting with the rostral side. The coordinate of the cross-sectional surface between Sections 7 and 8 corresponds to $1 \mathrm{~mm}$ anterior to the interaural line. Each section was incubated in a saline solution containing $2 \% 2,3,5$ triphenyltetrazolium chloride (Wako Pure Chemical Industries, Osaka, Japan) at $37{ }^{\circ} \mathrm{C}$ for $30 \mathrm{~min}$ and then lightly fixed by immersion in $10 \%$ formalin neutral buffer solution ( $\mathrm{pH} 7.4$, Wako Pure Chemical). The anterior surface of each section was photographed with a digital camera (Camedia C-1400XL, Olympus, Tokyo, Japan). The infarct area on the image of each slice was quantified with an image analyzer (Quantimet-600, Leica, Germany). The infarct volume in each slice was calculated from the area of infarction according to the following equation: $V=4\left\{a+b+(a b)^{1 / 2}\right\} / 3$, where $V$ is the infarct volume of a slice $\left(\mathrm{mm}^{3}\right), 4$ is the slice thickness $(\mathrm{mm}), a$ is the infarct area $\left(\mathrm{mm}^{2}\right)$, and $b$ is the infarct area of a slice caudal to the slice $\left(\mathrm{mm}^{2}\right)$. The infarct volume in the cerebral hemisphere, cortex, or striatum, was calculated by summing the sequential slice injury volumes. The analysis of lesion size was carried out under blinded conditions.

Statistical Analysis All data were expressed as mean values \pm S.D. Statistical analyses were performed by using EXSAS version 6.10 based on SAS release 8.2 (Arm Corp., Osaka, Japan, and SAS Institute Japan Ltd., Tokyo, Japan). Physiological parameters among the three groups were analyzed by two-way ANOVA followed by Dunnett's test. Infarct volume was analyzed by one-way ANOVA with Dunnett's test. Differences with a value of $p<0.05$ were considered statistically significant.

\section{RESULTS}

Physiological Parameters and EEG Appropriate monitoring of physiological parameters is essential to maintain the condition of experimental animals properly and to limit variability in infarct volume. As shown in Table 1, none of the physiological parameters, including blood gases, $\mathrm{pH}$, mean arterial blood pressure, and rectal temperature, showed significant differences among the groups over the time course of the experiments. MCA occlusion produced immediately a marked decrease in EEG amplitude, reaching less than 30\% of the preocclusion level (Fig. 1). There were no significant differences in the relative amplitudes before the start of DY9760e treatment among three experimental groups: vehicle, $13.7 \pm 4.3 \% ; 0.1 \mathrm{mg} / \mathrm{kg} / \mathrm{h}, 17.7 \pm 5.3 \% ; 0.25 \mathrm{mg} / \mathrm{kg} / \mathrm{h}, 19.5 \pm$ $9.5 \%$. This result implies the reduction in brain activities due to cerebral ischemia and indicates that animals could be ran-

Table 1. Effect of DY-9760e on Physiological Parameters in Cats Subjected to Permanent MCA Occlusion

\begin{tabular}{ccccc}
\hline \hline & $\begin{array}{c}\text { Infusion time } \\
\text { (h) }\end{array}$ & Vehicle & $0.1 \mathrm{mg} / \mathrm{kg} / \mathrm{h}$ & $0.25 \mathrm{mg} / \mathrm{kg} / \mathrm{h}$ \\
& 0 & $154.1 \pm 32.0$ & $159.9 \pm 15.2$ & $147.9 \pm 19.2$ \\
$\mathrm{PO}_{2}$ & 1 & $137.7 \pm 25.8$ & $142.7 \pm 21.1$ & $146.3 \pm 19.0$ \\
$(\mathrm{mmHg})$ & 3 & $136.1 \pm 31.8$ & $139.8 \pm 14.9$ & $143.5 \pm 17.5$ \\
& 6 & $138.0 \pm 27.7$ & $145.2 \pm 15.0$ & $140.5 \pm 21.3$ \\
& 0 & $30.4 \pm 0.6$ & $30.3 \pm 2.0$ & $31.5 \pm 2.6$ \\
$\mathrm{PCO}_{2}$ & 1 & $31.7 \pm 1.0$ & $31.6 \pm 2.2$ & $32.3 \pm 2.1$ \\
$(\mathrm{mmHg})$ & 3 & $33.6 \pm 2.1$ & $33.7 \pm 1.2$ & $34.4 \pm 1.6$ \\
& 6 & $31.6 \pm 1.5$ & $35.1 \pm 1.6$ & $33.9 \pm 2.1$ \\
& 0 & $7.38 \pm 0.02$ & $7.40 \pm 0.03$ & $7.35 \pm 0.03$ \\
$\mathrm{pH}$ & 1 & $7.38 \pm 0.02$ & $7.39 \pm 0.03$ & $7.35 \pm 0.03$ \\
& 3 & $7.36 \pm 0.03$ & $7.37 \pm 0.02$ & $7.33 \pm 0.03$ \\
& 6 & $7.38 \pm 0.02$ & $7.36 \pm 0.03$ & $7.34 \pm 0.02$ \\
$\mathrm{MABP}$ & 0 & $75.5 \pm 2.8$ & $76.7 \pm 2.0$ & $73.8 \pm 1.2$ \\
$(\mathrm{mmHg})$ & 1 & $76.2 \pm 2.6$ & $75.2 \pm 1.0$ & $75.5 \pm 2.6$ \\
& 3 & $73.5 \pm 3.2$ & $76.2 \pm 1.8$ & $75.3 \pm 2.1$ \\
& 6 & $73.5 \pm 2.2$ & $75.3 \pm 1.2$ & $72.0 \pm 0.9$ \\
$\mathrm{Rectal}$ & 0 & $36.8 \pm 0.15$ & $37.1 \pm 0.23$ & $36.9 \pm 0.23$ \\
temperature & 1 & $37.0 \pm 0.57$ & $37.0 \pm 0.24$ & $37.1 \pm 0.21$ \\
$\left({ }^{\circ} \mathrm{C}\right)$ & 3 & $37.1 \pm 0.06$ & $37.0 \pm 0.09$ & $37.0 \pm 0.16$ \\
& 6 & $37.1 \pm 0.18$ & $36.9 \pm 0.08$ & $37.1 \pm 0.20$ \\
\hline
\end{tabular}

Each value represents the mean \pm S.D. ( $n=6$ in each group). DY-9760e was infused intravenously starting $5 \mathrm{~min}$ after the onset of ischemia and continuing for $6 \mathrm{~h}$. 


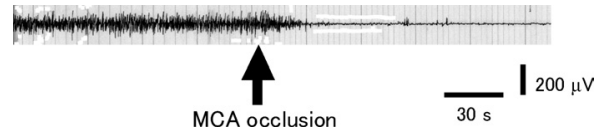

Fig. 1. Representative Record of EEG in Cats with a Permanent MCA Occlusion

The EEG was recorded on the surface of the bilateral middle ectosylvian gyri, where blood is supplied mainly by the MCA, during the experiment. The MCA was occluded at the point indicated by the arrow. The EEG amplitude was dramatically decreased by MCA occlusion.
A. Vehicle

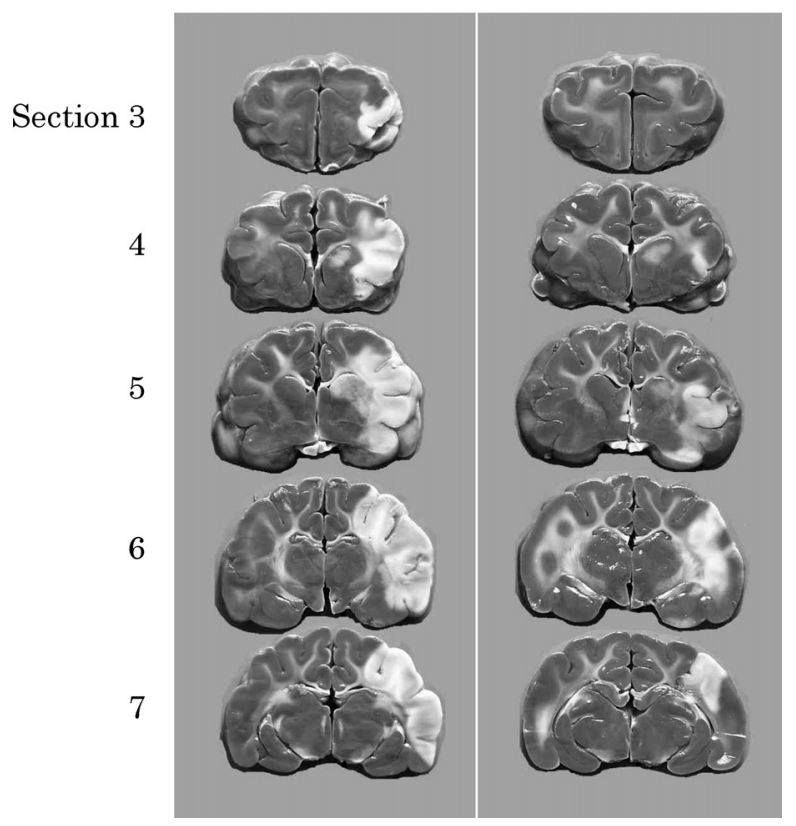

Fig. 2. Representative 2,3,5-Triphenyltetrazolium Chloride-Stained Coronal Brain Sections in Cats with Permanent MCA Occlusion

Each panel shows the photographs of Sections 3-7 in vehicle- (A) and DY-9760e $(0.25 \mathrm{mg} / \mathrm{kg} / \mathrm{h})$-treated groups (B). Infusion of vehicle or DY-9760e was started $5 \mathrm{~min}$ after MCA occlusion via the femoral vein for $6 \mathrm{~h}$.

domly divided into three groups on the basis of their relative amplitudes. The relative EEG amplitudes at the end of drug treatment were as follows: vehicle, $22.9 \pm 9.5 \% ; 0.1 \mathrm{mg} / \mathrm{kg} / \mathrm{h}$, $31.6 \pm 12.6 \% ; 0.25 \mathrm{mg} / \mathrm{kg} / \mathrm{h}, 42.5 \pm 28.7 \%$. Although statistical analysis showed no significant differences among the three groups, DY-9760e-treated animals had better recovery of EEG than those treated with vehicle. In preliminary works, we have confirmed that this compound has no effect on EEG in the side contralateral to the side of the lesion.

Effect of DY-9760e on Cerebral Infarct Volume The permanent MCA occlusion caused cerebral infarction in the MCA territories, including the entire striatum, where cerebral ischemia is most severe, and a region in the cortex called the penumbra, a treatable region (Fig. 2). Cats treated with DY-9760e $(0.25 \mathrm{mg} / \mathrm{kg} / \mathrm{h})$ had less infarction than did the vehicle-treated animals (Fig. 2). As shown in Fig. 3, DY-9760e, at a dose of $0.25 \mathrm{mg} / \mathrm{kg} / \mathrm{h}$ for $6 \mathrm{~h}$, significantly reduced the infarct volume by $57 \%$ in the cerebral hemisphere (from $2490 \pm 785 \mathrm{~mm}^{3}$ in vehicle-treated cats to $1082 \pm 729 \mathrm{~mm}^{3}$ in DY-9760e-treated cats) and by $58 \%$ in the cortex (from $2272 \pm 776 \mathrm{~mm}^{3}$ in vehicle-treated cats to $948 \pm 726 \mathrm{~mm}^{3}$ in DY-9760e-treated cats). In contrast, DY-9760e at this dose caused no significant reduction of infarct volume in the striatum, the ischemic core regions. The lower dose of

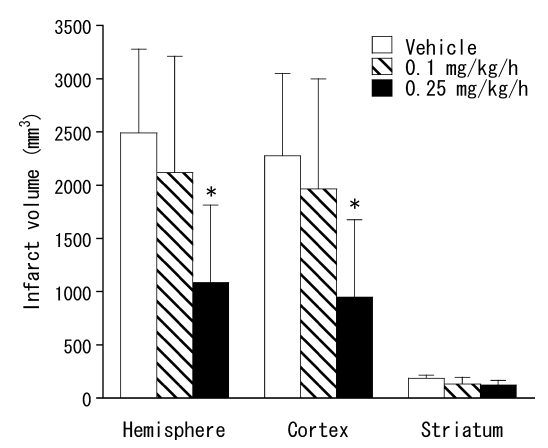

Fig. 3. Effect of DY-9760e on Cerebral Infarct Volume in Cats with Permanent MCA Occlusion

Data represent mean \pm S.D. ( $n=6$ in each group). $* p<0.05 v s$. vehicle (Dunnett's multiple comparison test).

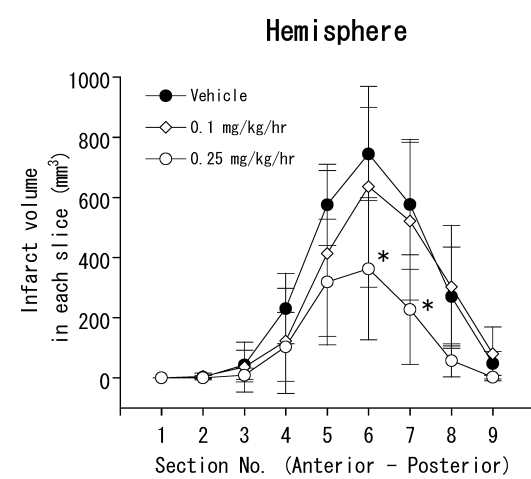

Fig. 4. Effect of DY-9760e on Infarct Volume in Each Coronal Section in Cats with the MCA Occlusion

Data represent mean \pm S.D. ( $n=6$ in each group). $* p<0.05 v s$. vehicle (Dunnett's multiple comparison test).

$0.1 \mathrm{mg} / \mathrm{kg} / \mathrm{h}$ for $6 \mathrm{~h}$ failed to reduce the infarct volume in any region. In comparison, in each coronal section, statistically significant decreases in infarction were detected in the Sections 6 and 7 (Fig. 4). These results suggest that the effective dose for this compound is $0.25 \mathrm{mg} / \mathrm{kg} / \mathrm{h}$ for $6 \mathrm{~h}$, and its protective effect is evident in the cortex, where the penumbra, a salvageable zone, exists.

\section{DISCUSSION}

Stroke is the third leading cause of death in major industrialized countries and also a major cause of long-lasting disability. At present, thrombolytic therapy with recombinant tissue plasminogen activator (rt-PA) is the only medication approved for the treatment of acute ischemic stroke. ${ }^{29)}$ However, the use of rt-PA is restricted to administration within 3 $h$ after onset of symptoms due to the risk of cerebral hemorrhage. ${ }^{30)}$ Therefore, there are strong clinical demands for neuroprotective agents that can block neuronal death associated with stroke. ${ }^{31)}$ We have developed DY-9760e, a calmodulin antagonist, which exerts neuroprotective action against cell death induced by $\mathrm{Ca}^{2+}$ overload ${ }^{15,17)}$ and ameliorates brain injury that occurs after cerebral ischemia in rodents. $^{18-22,32,33)}$ The present study demonstrates that DY9760e reduces the infarct volume in cats with permanent MCA occlusion, and this finding is consistent with previous studies in rats with permanent MCA occlusion, extending the observation into a gyrencephalic species. ${ }^{20,21)}$ 
Focal ischemia models can be categorized mainly into two types: permanent and transient. Although both types of focal ischemia can occur in stroke patients, clinically the majority of strokes do not reperfuse in the first $24 \mathrm{~h} .{ }^{34)}$ Furthermore, the Stroke Therapy Academic Industry Roundtable stated that novel compounds should show efficacy in species larger than rodents. ${ }^{35)}$ We therefore evaluated the effect of DY$9760 \mathrm{e}$ on brain injury by using a cat model with permanent MCA occlusion. Treatment with DY-9760e, when administered at the rate of $0.25 \mathrm{mg} / \mathrm{kg} / \mathrm{h}$, resulted in a significant reduction in the infarct volume without affecting any physiological parameters. The reduction was pronounced in the cortex, where the penumbra exists, but not in the striatum. Previous evidence that during ischemia, the striatum has the most severe reduction in blood flow followed by rapid cell death may explain why DY-9760e failed to rescue the striatum from ischemic damage. ${ }^{36-39)}$

It is known that the degree of EEG amplitude reduction following ischemic insult is associated with a significant reduction in cerebral blood flow $(\mathrm{CBF}){ }^{40)}$ In addition, there is evidence to suggest a close correlation between the extent of brain injury and EEG changes in focal ischemia models. ${ }^{41,42)}$ We also observed a pronounced decrease in EEG immediately after MCA occlusion, resulting from the reduction in brain activities due to the cerebral ischemia. Interestingly, animals receiving DY-9760e tended to have better recovery of EEG than those treated with vehicle. This result supports the notion that DY-9760e exerts a neuroprotective effect against cerebral ischemia. Considering the fact that DY-9760e does not alter CBF following MCA occlusion in rats, ${ }^{21)}$ however, its neuroprotection may be mediated through mechanisms other than the improvement of CBF. Indeed, DY-9760e can rescue neurons from cell death elicited by cell-toxic stimuli that mimic cellular events observed in cerebral ischemia. ${ }^{15,17)}$ In addition, it has been shown that trifluoperazine, a calmodulin antagonist that is structurally different from DY-9760e, attenuates brain injury after transient MCA occlusion without improving $\mathrm{CBF}^{43)}$ Taken together, these results indicate that the neuroprotection demonstrated in animal models may be due to a direct action of DY-9760e in the brain parenchyma.

It is widely accepted that an excessive elevation of intracellular $\mathrm{Ca}^{2+}$ concentration plays a major role in ischemic cell injury in neurons. ${ }^{1-3)}$ The elevated intracellular $\mathrm{Ca}^{2+}$, even that by any route, can bind to $\mathrm{Ca}^{2+}$-binding proteins, including calmodulin. There is evidence that calmodulin plays a critical role in ischemic brain injury: (1) a persistent increase of $\mathrm{Ca}^{2+}$-bound calmodulin, which is an active form, after ischemic insults; (2) significant up-regulation of calmodulin gene expression in the CA1 pyramidal cell layer after cerebral ischemia; and (3) protection by calmodulin antagonists against hypoxic/hypoglycemia in organotypic hippocampal cultures. ${ }^{12,14,44)}$ Furthermore, there are reports that cells expressing PEP-19, a brain-specific negative regulator of calmodulin, or a mutant calmodulin with $\mathrm{Ca}^{2+}$-binding defects are resistant to apoptotic stimuli. ${ }^{45,46)}$ DY-9760e rescues cultured neurons from a wide variety of cell-toxic stimuli that increase intracellular $\mathrm{Ca}^{2+}$, including excitotoxicity, voltage-gated channel opening, $\mathrm{Ca}^{2+}$ ionophore, and inhibition of endoplasmic reticulum $\mathrm{Ca}^{2+}$-ATPase. ${ }^{15,17)}$ DY-9760e is also capable of reducing the ischemic infarct volume when given up to $3 \mathrm{~h}$ after the onset of permanent MCA occlusion in rats. ${ }^{20)}$ Given a previous report showing that the $\mathrm{Ca}^{2+}$ bound calmodulin complex reaches maximal levels $4 \mathrm{~h}$ following cerebral ischemia, it is feasible that the therapeutic time window for DY-9760e is $3 \mathrm{~h}^{12}{ }^{12}$ Taken together, it is plausible that the amelioration of ischemic brain injury with this drug is mediated by a calmodulin antagonistic action in the brain. Further studies are needed to evaluate the molecular mechanism by which this compound exerts neuroprotective functions.

In conclusion, DY-9760e reduces the cerebral infarct volume in cats with permanent MCA occlusion. Therefore, drugs that inhibit aberrant activation of calmodulin may provide therapeutic value in the treatment of acute stroke.

Acknowledgements The authors thank Yoshio Kasai and Naoko Edo for their technical assistance with measurements of EEG and infarct volume.

\section{REFERENCES}

1) Choi D. W., Trends Neurosci., 18, 58-60 (1995).

2) Meldrum B., Garthwaite J., Trends Pharmacol. Sci., 11, 379-387 (1990).

3) Mitani A., Yanase H., Sakai K., Wake Y., Kataoka K., Brain Res., 601, 103-110 (1993).

4) Sauer D., Allegrini P. R., Cosenti A., Pataki A., Amacker H., Fagg G. E., J. Cereb. Blood Flow Metab., 13, 595-602 (1995).

5) Horn J., de Haan R. J., Vermeulen M., Luiten P. G. M., Limberg M., Stroke, 32, 2433-2438 (2001).

6) Schäbitz W.-R., Li F., Fischer M., Stroke, 31, 1709-1714 (2000).

7) Davis S. M., Lees K. R., Albers G. W., Diener H. C., Markabi S., Karlsson G., Norris J., Stroke, 31, 347-354 (2000).

8) Horn J., Limburg M., Stroke, 32, 570—576 (2001).

9) Albers G. W., Goldstein L. B., Hall D., Lesko L. M., J. Am. Med. Assoc., 286, 2673-2682 (2001).

10) Zhou L. W., Moyer J. A., Muth E. A., Clark B., Palkovits M., Weiss B., J. Neurochem., 44, 1657-1662 (1985).

11) James P., Vorherr T., Carafoli E., Trends Biochem. Sci., 20, 38-42 (1995).

12) Picone C. M., Grotta J. C., Earls R., Strong R., Dedman J., J. Cereb. Blood Flow Metab., 9, 805-811 (1989).

13) Dawson V. L., Dawson T. M., Bartley D. A., Uhl G. R., Snyder S. H., J. Neurosci., 13, 2651-2661 (1993).

14) Sun X., Shin C., Windebank A. J., Neuroreport, 8, 415-418 (1997).

15) Sugimura M., Sato T., Nakayama W., Morishima Y., Fukunaga K., Omitsu M., Miyamoto E., Shirasaki Y., Eur. J. Pharmacol., 336, 99 106 (1997).

16) Fukunaga K., Ohmitsu M., Miyamoto E., Sato T., Sugimura M., Uchida T., Shirasaki Y., Biochem. Pharmacol., 60, 693-699 (2000).

17) Takano H., Sugimura M., Kanazawa Y., Uchida T., Morishima Y., Shirasaki Y., Biol. Pharm. Bull., 27, 1788-1791 (2004).

18) Sato T., Morishima Y., Sugimura M., Uchida T., Shirasaki Y., Eur. J. Pharmacol., 370, 117-123 (1999).

19) Sato T., Morishima Y., Shirasaki Y., J. Pharmacol. Exp. Ther, 304, 1042-1047 (2003)

20) Sato T., Takamori H., Shirasaki Y., Pharmacology, 71, 38 - 45 (2004).

21) Takagi K., Sato T., Shirasaki Y., Narita K., Tamura A., Sano K., Neurol. Res., 23, 662-668 (2001).

22) Shirasaki Y., Edo N., Sato T., Brain Res., 1021, 159-166 (2004).

23) Bullock R., Graham D. I., Chen M.-H., Lowe D., McCulloch J., J. Cereb. Blood Flow Metab., 10, 668-674 (1990).

24) Patel T. R., Galbraith S., Graham D. I., Hallak H., Doherty A. M., McCulloch J., J. Cereb. Blood Flow Metab., 16, 950-958 (1996).

25) Akiho H., Iwai A., Tsukamoto S., Koshiya K., Yamaguchi T., Neuropharmacology, 37, 159-168 (1998).

26) Takahashi M., Ni J. W., Kawasaki-Yatsugi S., Toya T., Yatsugi S., J. Pharmacol. Exp. Ther., 284, 467-473 (1998).

27) Heiss W.-D., Graf R., Wienhard K., Cerebrovasc. Dis., 11, 73-81 
(2001).

28) O’Brien M. D., Waltz A. G., Stroke, 4, 201-206 (1973).

29) Fagan S. C., Morgenstern L. B., Petitta A., Ward R. E., Tilley B. C., Marler J. R., Levine S. R., Broderick J. P., Kwiatkowski T. G., Frankel M., Brott T. G., Walker M. D., Neurology, 50, 883-890 (1998).

30) Bravata D. M., Kim N., Concato J., Krumholz H. M., Brass L. M., Arch. Intern. Med., 162, 1994-2001 (2002).

31) Stapf C., Mohr J. P., Annu. Rev. Med., 53, 453- 475 (2002).

32) Hashiguchi A., Kawano T., Yano S., Morioka M., Hamada J., Sato T., Shirasaki Y., Ushio Y., Fukunaga K., Neuroscience, 121, 379-386 (2003).

33) Hashiguchi A., Yano S., Morioka M., Hamada J., Shirasaki Y., Kochi M., Fukunaga K., J. Pharmacol. Sci., 96, 65-72 (2004).

34) Minematsu K., Yamaguchi T., Omae T., Neurology, 42, 157-162 (1992).

35) Stroke Therapy Academic Industrial roundtable (STAIR), Stroke, 30, 2752-2758 (1999).

36) DeGraba T. J., Ostrow P. T., Grotta J. C., Stroke, 24, 1212-1216; discussion 1216-1217 (1993).
37) Fisher M., Takano K., Baillieres Clin. Neurol., 4, 279-295 (1995).

38) Memezawa H., Minamisawa H., Smith M. L., Siesjo B. K., Exp. Brain Res., 89, 67-78 (1992).

39) Reith W., Hasegawa Y., Latour L. L., Dardzinski B. J., Sotak C. H., Fisher M., Neurology, 45, 172-177 (1995).

40) McGrail K. M., Neurosurg. Clin. N. Am., 7, 685-692 (1996).

41) Williams A. J., Lu X.-C. M., Hartings J. A., Tortella F. C., Fundam. Clin. Pharmacol., 17, 581-593 (2003).

42) Yatsugi S., Takahashi M., Kawasaki-Yatsugi S., Koshiya K., Sakamoto S., Uematsu D., Shimizu-Sasamata M., J. Cereb. Blood Flow Metab., 16, 959-966 (1996).

43) Kuroda S., Nakai A., Kristian T., Siesjö B. K., Stroke, 28, 2539—2544 (1997).

44) Palfi A., Simonka J. A., Pataricza M., Tekulics P., Lepran I., Papp G., Gulya K., Life Sci., 68, 2373-2381 (2001).

45) Erhardt J. A., Legos J. J., Johanson R. A., Slemmon J. R., Wang X., Neuroreport, 11, 3719-3723 (2000).

46) Schmalzigaug R., Ye Q., Berchtold M. W., Immunology, 103, 332342 (2001). 